\title{
Binding, Domain Orientation, and Dynamics of the Lck SH3-SH2 Domain Pair and Comparison with Other Src-Family Kinases ${ }^{\dagger}$
}

\author{
Gregor Hofmann, ${ }^{\ddagger}$ Kristian Schweimer, ${ }^{\S}$ Anke Kiessling, ${ }^{\S}$ Edith Hofinger,${ }^{\S}$ Finn Bauer,${ }^{\S, l}$ Silke Hoffmann, ${ }^{\S}$ \\ Paul Rösch, ${ }^{\S}$ Iain D. Campbell, ${ }^{\ddagger}$ Jörn M. Werner, ${ }^{\ddagger}, \perp$ and Heinrich Sticht*,ll \\ Department of Biochemistry, University of Oxford, South Parks Road, Oxford OX1 3QU, U.K., Lehrstuhl für Biopolymere, \\ Universität Bayreuth, D-95440 Bayreuth, Germany, and Institut für Biochemie, Emil-Fischer-Zentrum, \\ Universität Erlangen-Nürnberg, D-91054 Erlangen, Germany
}

Received May 3, 2005; Revised Manuscript Received August 1, 2005

\begin{abstract}
The catalytic activity of Src-family kinases is regulated by association with its SH3 and SH2 domains. Activation requires displacement of intermolecular contacts by $\mathrm{SH} 3 / \mathrm{SH} 2$ binding ligands resulting in dissociation of the SH3 and SH2 domains from the kinase domain. To understand the contribution of the $\mathrm{SH} 3-\mathrm{SH} 2$ domain pair to this regulatory process, the binding of peptides derived from physiologically relevant $\mathrm{SH} 2$ and $\mathrm{SH} 3$ interaction partners was studied for Lck and its relative Fyn by NMR spectroscopy. In contrast to Fyn, activating ligands do not induce communication between $\mathrm{SH} 2$ and $\mathrm{SH} 3$ domains in Lck. This can be attributed to the particular properties of the Lck SH3-SH2 linker which is shown to be extremely flexible thus effectively decoupling the behavior of the $\mathrm{SH} 3$ and $\mathrm{SH} 2$ domains. Measurements on the SH32 tandem from Lck further revealed a relative domain orientation that is distinctly different from that found in the Lck SH32 crystal structure and in other Src kinases. These data suggest that flexibility between $\mathrm{SH} 2$ and $\mathrm{SH} 3$ domains contributes to the adaptation of Src-family kinases to specific environments and distinct functions.
\end{abstract}

Src family tyrosine kinases are implicated in a large number of cellular processes including cell adhesion and spreading, focal adhesion formation and disassembly, cell migration, cell cycle progression, apoptosis, cell differentiation, and gene transcription $(1,2)$. Mutations in these proteins can also lead to cancer (3). All members of the family (Src, Blk, Fgr, Fyn, Hck, Lck, Lyn, Yes, Yrk) have a common molecular architecture that includes regulatory $\mathrm{SH} 3{ }^{1}$ and $\mathrm{SH} 2$ domains and a kinase domain. In its inactive state, the kinase is inhibited by binding of the $\mathrm{SH} 2$ domain to a conserved tyrosine in the $\mathrm{C}$-terminal regulatory tail and by binding of the SH3 domain to the SH2-kinase linker region (4-6). Dephosphorylation of this conserved tyrosine (7) or binding of competing ligands to the $\mathrm{SH} 2$ or SH3 domains (8) activates the kinase. Young et al. (9) suggested that coupling between $\mathrm{SH} 2$ and $\mathrm{SH} 3$ is important in controlling activation, because mutations of residues in the $\mathrm{SH} 3-\mathrm{SH} 2$ linker

\footnotetext{
This work was supported by grants of the Deutsche Forschungsgemeinschaft (SFB466: A5, C11) to P.R. and H.S. and by the Wellcome Trust to I.D.C. and J.M.W.

* To whom correspondence should be addressed. Tel: +49 9131 8524614. Fax: +49 9131 8522485. E-mail: H.Sticht@biochem.uni-erlangen.de.

$\doteqdot$ University of Oxford.

$\S$ Universität Bayreuth.

" Universität Erlangen-Nürnberg.

${ }^{\perp}$ Present address: School of Biological Sciences, University of Southampton, Southampton SO16 7PX, U.K.

${ }^{1}$ Abbreviations: HMTA, hamster polyomavirus middle T-antigen; HSQC, heteronuclear single-quantum coherence; NMR, nuclear magnetic resonance; PAG, polyacrylamide gel; RDC, residual dipolar coupling; RMSD, root-mean-square deviation; SH2, Src-homology domain 2; SH3, Src-homology domain 3; SH32, SH3-SH2 domain pair; Tip, tyrosine kinase interacting protein
}

activated c-Src. Molecular dynamics simulations also suggested that, in the inactive state, the coupling between the $\mathrm{SH} 3-\mathrm{SH} 2$ dynamics was tight, and that this no longer applies when the regulatory tail is detached from the $\mathrm{SH} 2$ domain (9). Hence, they proposed that the $\mathrm{SH} 3-\mathrm{SH} 2$-kinase assembly represented an "inducible snap lock".

While the crystal structures of c-Src and Hck have provided a wealth of structural information on the closed, inactive state of Src-kinases, the open state is much less well characterized, in terms of both structure and dynamics. Crystal structures have been obtained for the $\mathrm{SH} 3-\mathrm{SH} 2$ domain pairs of Lck (10) and Fyn (11). These fragments might be expected to behave in a similar way to the domains when they are detached from the kinase in the activated state. There has, therefore, been some previous interest in studying the solution behavior of $\mathrm{SH} 3-\mathrm{SH} 2$ domain pairs including those of Src (12), Abl (13), and Fyn (14). Comparison of the domain orientation observed in the Fyn SH32 crystal structure with the domain orientation of peptide bound Fyn SH32 observed in solution showed a relatively modest average domain reorientation along one rotation axis. This is consistent with a situation that is intermediate between a rigid and a completely decoupled interdomain interface (14). Intriguingly, the orientation between the $\mathrm{SH} 3$ and $\mathrm{SH} 2$ domains in the crystal structure of Lck SH32 is very different from that observed for other Src-family members. Here solution NMR spectroscopy is used to determine the domain orientation of Lck SH32 in solution and to characterize the dynamics of this domain pair. Binding of peptides derived from physiologically relevant ligands to both Fyn SH32 and Lck SH32 is also investigated to provide further insight into 
the contribution made by $\mathrm{SH} 3-\mathrm{SH} 2$ domain pairs to the regulation of Src kinases.

\section{MATERIALS AND METHODS}

Protein Production and Peptide Ligands. Expression and purification of the SH3-SH2 domain pairs of human Lck (Lck SH32; residues 57-225) and Fyn (Fyn SH32; residues 81-247 with C239S, C240S, and C246S) were performed as described previously $(11,14,15)$. Minimal medium with ${ }^{15} \mathrm{NH}_{4} \mathrm{Cl}$ and if necessary $\mathrm{D}_{2} \mathrm{O}$ instead of $\mathrm{H}_{2} \mathrm{O}$ was used to produce ${ }^{15} \mathrm{~N}$-labeled Lck SH32 and ${ }^{15} \mathrm{~N},{ }^{2} \mathrm{H}$-labeled Fyn SH32. Purity of the sample was confirmed by SDS-polyacrylamide gel electrophoresis, and concentration was assessed by the optical density at $280 \mathrm{~nm}$, using extinction coefficients calculated from the amino acid sequence.

Peptide ligands for $\mathrm{SH} 3$ and $\mathrm{SH} 2$ binding experiments were purchased from Biosyntan (Berlin, Germany) and Coring (Gernsheim, Germany) and contained blocked end groups. The Tip peptide comprises residues 167-199 of the tyrosine-kinase interacting (Tip) protein from Herpesvirus saimiri strain C488 and the phosphorylated HMTA peptide the $\mathrm{CQ}(\mathrm{pY}) \mathrm{EEIP}$ sequence from the middle $\mathrm{T}$ antigen of hamster polyomavirus. The $\mathrm{P} \rightarrow \mathrm{C}$ point mutation at the $\mathrm{N}$-terminal sequence position of the investigated peptide was shown to have no effect on $\mathrm{SH} 2$ binding affinity (Hofinger and Sticht, unpublished).

NMR Sample Preparation and Data Acquisition. Protein solutions were prepared in the following buffer conditions: $100 \mathrm{mM} \mathrm{K} 2 \mathrm{HPO}_{4} / \mathrm{KH}_{2} \mathrm{PO}_{4}$, pH 6.5, $20 \mathrm{mM} \mathrm{NaCl}$ for Lck SH32 and $50 \mathrm{mM} \mathrm{K} \mathrm{HPO}_{4} / \mathrm{KH}_{2} \mathrm{PO}_{4}$, pH 7.0, $200 \mathrm{mM} \mathrm{Na}_{2}-$ $\mathrm{SO}_{4}$ for Fyn $\mathrm{SH} 32$. For the peptide titrations, aliquots of peptide of known concentration were first dried using a Speed-Vac apparatus and successive aliquots were then dissolved in the sample solution.

Polyacrylamide gels were prepared as described by Chou et al. (16) and were squeezed into an open-ended NMR tube by application of pressure, using a home-built squeezer. For recording residual dipolar couplings in liquid crystalline medium, a sample of $450 \mu \mathrm{M}$ Lck SH32 in monododecylpentaethyleneglycol-ether/hexanol (molar ratio $0.95 ; 3 \%$ (wt) $\mathrm{C} 12 \mathrm{E} 5 / \mathrm{H}_{2} \mathrm{O}$ ) was used that was prepared as described in Rückert \& Otting (17). For all systems, proper alignment was checked by measuring the quadrupolar splitting of the deuterium resonance of $\mathrm{D}_{2} \mathrm{O}$. NMR experiments were performed on spectrometers operating at ${ }^{1} \mathrm{H}$ frequencies of 500, 600, and $750 \mathrm{MHz}$. Unless otherwise stated, all spectra were recorded at $25{ }^{\circ} \mathrm{C}$. Backbone resonance assignments of Lck SH32 and Fyn SH32 have been described previously $(11,15)$.

Measurement and Interpretation of Relaxation Data. Twodimensional $\left\{{ }^{1} \mathrm{H}\right\}-{ }^{15} \mathrm{~N}$ heteronuclear NOE experiments and a series of ${ }^{1} \mathrm{H}-{ }^{15} \mathrm{~N}$ correlation spectra for the determination of ${ }^{15} \mathrm{~N} T_{1}$ and $T_{2}$ relaxation time constants were acquired using previously described methods incorporating pulsed field gradients for coherence pathway selection and water suppression $(18-20) .{ }^{15} \mathrm{~N}-T_{2}$ constants were measured using a spin-echo sequence with a Carr-Purcell-Meiboom-Gill (CPMG) delay of $419.4 \mu \mathrm{s}$. Dipolar and chemical shift anisotropy (CSA) cross-correlation occurring during delay periods were removed by applying ${ }^{1} \mathrm{H} 180^{\circ}$ pulses. This was performed once in the middle of the CPMG delay block for
${ }^{15} \mathrm{~N}-T_{2}$ experiments and every $5 \mathrm{~ms}$ in the delay period of ${ }^{15} \mathrm{~N}-T_{1}$ experiments $(21,22)$. The total sample heating for different experiments was equalized by application of a "heat" pulse train to keep the total number of ${ }^{15} \mathrm{~N} 180^{\circ}$ pulses constant for all experiments. Each series of $T_{1}$ and $T_{2}$ measurements consisted of 8 autocorrelation spectra with increasing ${ }^{15} \mathrm{~N}$ relaxation time delays, chosen to sample approximately the entirety of the observed intensity decays. All spectra were processed with mild resolution enhancement and linear prediction in the indirect dimension using Felix 2.3 (Biosym, San Diego, CA). After zero filling, the digital resolution of the spectra was $6.1 \mathrm{~Hz} /$ point in the ${ }^{1} \mathrm{H}$ dimension and $1.3 \mathrm{~Hz} /$ point in the ${ }^{15} \mathrm{~N}$ dimension. Relaxation parameters were estimated from two parameter exponential fits to the intensity decays in the series of $T_{1} T_{2}$ correlation spectra. Errors were estimated using the baseline noise (23).

To test initially for the aggregation state of free Lck SH32, experimental $T_{1}$ and $T_{2}$ relaxation times were compared with calculated relaxation times as a function of isotropic correlation time and order parameter $\left(S^{2}\right)$ using the Lipari and Szabo model (24). For Lck SH32 correlation times and diffusion tensors were derived using the $T_{1}$ and $T_{2}$ values of residues that are part of secondary structure elements and whose values were inside the $S^{2}=1$ envelope of the LipariSzabo model. The principal axes, $D_{x}, D_{y}$, and $D_{z}$, of the diffusion tensor were determined by global least-squares fits of the $T_{1} / T_{2}$ ratios derived from the spectral density functions of a diffusing particle to the experimental values (25-27). Three models of increasing complexity were tested: a sphere [ $\left.D_{x}=D_{y}=D_{z}, D=\left(D_{x}+D_{y}+D_{z}\right) / 3\right]$, a symmetric top $\left[D_{\|}=D_{z}\right.$, and $\left.D_{\perp}=\left(D_{x}+D_{y}\right) / 2\right]$, and a fully asymmetric tensor $\left(D_{x} \neq D_{y} \neq D_{z}\right)(28)$. The analysis was performed with programs written in-house. The NH bond length of backbone amides was assumed to be $1.02 \AA$, and the chemical shift anisotropy (CSA) was assumed to be -170 ppm with the CSA tensor taken to be collinear with the dipolar vector. Model comparisons were performed by using the $F$-test and calculation of the probability $Q$ to obtain this $F$ by chance (29). Generally, $Q$ values below $5 \%$ were assumed to justify the use of the more complex model.

Domain Orientation from Residual Dipolar Couplings. Apparent $J_{\mathrm{NH}}$ constants of ${ }^{15} \mathrm{~N}$-labeled Lck SH32 were obtained using the in-phase, antiphase (IPAP) scheme (30). Two sets of data were collected at temperatures of $25^{\circ} \mathrm{C}$ and $20{ }^{\circ} \mathrm{C}$ from an aligned gel sample and a sample containing a liquid crystalline phase (as described above), respectively. All spectra were processed with resolution enhancement in both ${ }^{15} \mathrm{~N}$ and ${ }^{1} \mathrm{H}$ dimensions and linear prediction in the indirect dimension using Felix 2.3. After zero filling the digital resolution of the spectra was 0.45 and $7.81 \mathrm{~Hz}$ for the ${ }^{15} \mathrm{~N}$ and ${ }^{1} \mathrm{H}$ dimensions, respectively.

The residual dipolar ${ }^{15} \mathrm{~N}-{ }^{1} \mathrm{H}$ coupling (RDC) was calculated from the apparent $J_{\mathrm{NH}}$ constants for spectra collected for the aligned and isotropic media: $\mathrm{RDC}=J_{\mathrm{NH} \text {,aligned }}-$

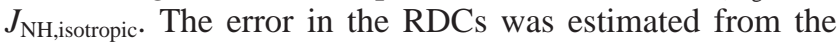
ratios of the line widths and signal-to-noise values in each spectrum using error propagation. Nonlinear optimization of the fit of the experimental residual dipolar couplings to calculated values, as a function of alignment tensor parameters, was performed using the program MODULE (31) and routines written in-house. Only dipolar couplings from residues in regular secondary structure elements were used 
for analysis. Best fits were determined by $\chi^{2}$ minimization, where $\chi^{2}$ is defined as

$$
\chi^{2}=\sum_{i=0}^{N} \frac{\left(\mathrm{RDC}_{\text {experiment }, i}-\mathrm{RDC}_{\text {simulated }, i}\right)^{2}}{\sigma^{2}}
$$

$\sigma^{2}$ was taken to be the error in the measured RDCs, estimated to be $2.0 \mathrm{~Hz}$ from the ratios of the line width and signalto-noise values in each spectrum using error propagation.

Ligand Binding Studies. The binding of Tip(167-199) and HMTA to Lck SH32 and Fyn SH32 was monitored by chemical shift perturbations in a series of ${ }^{1} \mathrm{H}-{ }^{15} \mathrm{~N}$ HSQC experiments upon titration of unlabeled ligand to the ${ }^{15} \mathrm{~N}$ labeled SH32 domains. All titrations were performed to an at least 4-fold excess of ligand. Eight spectra with Tip peptide concentrations ranging from 0 to $1.72 \mathrm{mM}$ were collected for experiments on Fyn SH32, and the corresponding protein concentration was $250 \mu \mathrm{M}$. Binding constants for Tip and Fyn SH32 were estimated from chemical shift changes of individual nuclei at different peptide concentrations by nonlinear curve fitting (32) according to the equation

$$
\Delta=\Delta_{0} \frac{K_{\mathrm{d}}+[\mathrm{L}]+[\mathrm{P}]-\sqrt{\left(K_{\mathrm{d}}+[\mathrm{L}]+[\mathrm{P}]\right)^{2}-4[\mathrm{~L}][\mathrm{P}]}}{2[\mathrm{P}]}
$$

where $\Delta$ is the chemical shift change, $\Delta_{0}$ is the chemical shift change at saturation, $K_{\mathrm{d}}$ is the binding constant, and $[\mathrm{L}]$ and $[\mathrm{P}]$ are the concentrations of peptide ligand and protein, respectively. This formula assumes a simple [protein] + [ligand $] \rightleftarrows[$ complex] binding model. An estimate of the binding constant $K_{\mathrm{d}}$ for the entire complex was made as the average of the measured $K_{\mathrm{d}}$ values for individual nuclei. The error in $K_{\mathrm{d}}$ was estimated as the standard deviation of the sampled $K_{\mathrm{d}}$ values.

Since intermediate and slow exchange phenomena on the NMR time scale were observed for several resonances in the Tip(167-199)-Lck SH32 titration experiment, the binding constant was determined by fluorescence measurements in an identical fashion as described for Tip(168-187) and Lck SH3 (33). The W170L point mutation present in the Tip peptides used for fluorescence measurements was previously shown to have no effect on the Tip-Lck affinity (33). Since the concentration of Lck SH32 was always low compared to the ligand concentration, the experimental data were fitted to the equation $F=F_{\max }[\mathrm{L}] /\left(K_{\mathrm{d}}+[\mathrm{L}]\right)$, where $[\mathrm{L}]$ is the final ligand concentration at each measurement point, $F$ is the measured protein fluorescence intensity at the particular peptide concentration, and $F_{\max }$ is the observed maximal fluorescence intensity of the protein when saturated with the peptide. Nonlinear regression curve fitting was carried out to fit the experimental data to the equation, with $F_{\max }$ and $K_{\mathrm{d}}$ as fitted parameters. The change in protein concentration that occurred as a result of peptide addition was properly corrected.

\section{RESULTS AND DISCUSSION}

SH3 and SH2 Domain Communication by Ligand-Binding to Lck SH32 and Fyn SH32. Two peptides that are specific for $\mathrm{SH} 3$ and $\mathrm{SH} 2$ domain binding respectively were used to investigate the effect on interdomain communication in Lck SH32 and Fyn SH32.
The herpesviral tyrosine kinase interacting protein (Tip) contains a proline-rich sequence [Tip(167-199)] that binds to the SH3 domains of several Src-family kinases (33), and this interaction was shown to be sufficient for a moderate activation of Lck (34). Importantly, Fyn is not activated by Tip (35) even though both Lck and Fyn are expressed in T-lymphocytes.

HMTA is derived from the middle T-antigen of hamster polyomavirus. It contains a phosphotyrosine site and is the strongest Lck SH2 ligand known $\left(K_{\mathrm{d}} \sim 140 \mathrm{nM}(36-38)\right)$. HMTA activates a range of Src-family kinases including both Lck and Fyn (39-42).

Chemical shift changes induced by titrating a protein solution with a ligand were used to identify local environmental changes caused by complex formation. With respect to domain-domain interactions, chemical shift changes in the domain that is not involved in direct binding of the respective ligand are of particular interest, because these indicate possible propagation of structural changes from one domain to the other. Such changes were indeed found for the interaction of the SH32 domain pairs of Src (12) and Fyn (14) with peptides derived from natural ligands. Communication of this kind between domains was suggested to be important in the activation of Src-kinases $(9,14)$ and may be involved in kinase regulation.

Tip(167-199) binding to Lck SH32 and Fyn SH32 was monitored by changes in ${ }^{1} \mathrm{H}$ and ${ }^{15} \mathrm{~N}$ chemical shifts of the proteins. The dissociation constant, $K_{\mathrm{d}}$, for the Tip(167199):Lck SH32 complex was determined to be $3.8 \pm 0.2$ $\mu \mathrm{M}$ using fluorescence spectroscopy. Both the chemical shift changes and the $K_{\mathrm{d}}$ are similar to the results for the interaction of an almost identical Tip-peptide with the Lck SH3 domain alone (33). No chemical shift changes could be observed in the SH2 domain. Hence, Tip(167-199) binding to the SH3 domain of Lck SH32 does not appear to induce structural changes in the $\mathrm{SH} 2$ domain of this domain pair (Figure 1a).

The dissociation constant of the Tip(167-199):Fyn SH32 complex was $64 \pm 18 \mu \mathrm{M}$. This affinity is about 15 times lower than for the Tip(167-199):Lck SH32 complex and about 2-fold lower than for a focal adhesion kinase derived SH3 ligand that activates Fyn (14). Only one noticeable chemical shift change is found in the $\mathrm{SH} 2$ domain (residue Leu163). Thus, generally no structural change seems to be transmitted through the SH3-SH2 linker upon Tip(167199) binding (Figure 1a,b). Since such changes were typically observed in experiments with ligands that do activate Fyn (14), communication between domains may be a critical feature of natural partners of Fyn. This feature is not shared by $\operatorname{Tip}(167-199)$.

The interdomain communication in Lck SH32 was further investigated by monitoring the chemical shift changes upon titration of the SH2 specific ligand HMTA. This did not lead to any chemical shift changes in the SH3 domain, suggesting the absence of structural communication between domains even for this very high affinity Lck SH2 ligand (Figure 1c). In contrast, binding of HMTA to both Src SH32 (12) and Fyn SH32 (14) did confer chemical shift changes in the respective SH3 domains. This suggests that Lck differs from other Src-kinases in that it has no facility for structural communication through the $\mathrm{SH} 3-\mathrm{SH} 2$ linker, possibly 

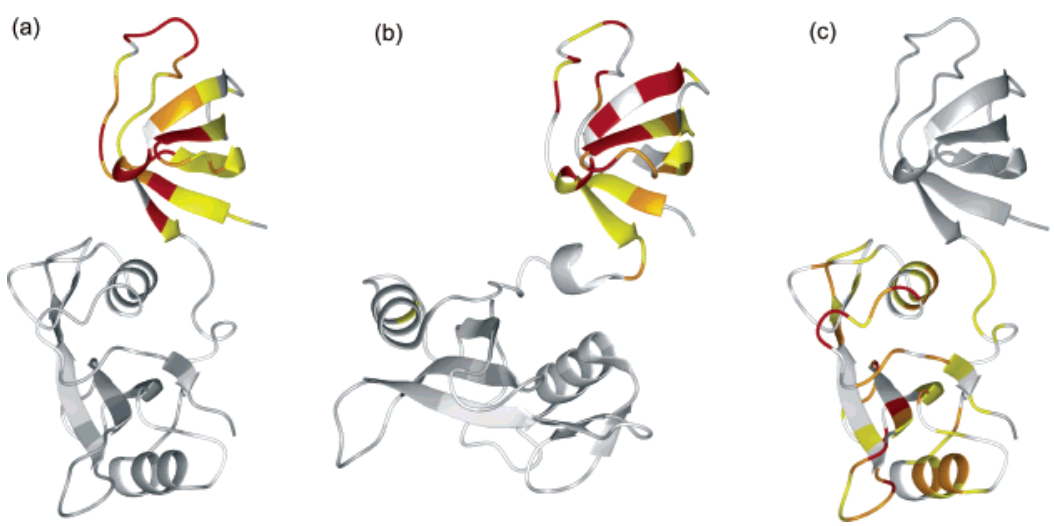

FIGURE 1: (a) Interaction of Lck SH32 with the Tip(167-199) peptide that binds to the SH3 domain. Residues are colored in red, orange, and yellow if the magnitude of the changes of the normalized chemical shift upon titration exceeds $0.12,0.06$, or 0.04 ppm, respectively. Residues for which insignificant changes of the normalized chemical shifts $(<0.04 \mathrm{ppm})$ were detected or for which no shift data could be obtained are colored in gray and white, respectively. All normalized values of the chemical shifts given were calculated as $\Delta_{\text {norm }}=\left[\left(\Delta_{\mathrm{HN}}\right)^{2}\right.$ $\left.\left.+\left(\Delta_{N} / 10\right)^{2}\right)\right]^{1 / 2}$. (b) Ribbon diagram of the crystal structure of Fyn SH32 showing chemical shift changes on Tip(167-199) binding. Residues are colored in red, orange, and yellow if the magnitude of the changes of the normalized chemical shift upon titration exceeds $0.20,0.10$, or $0.04 \mathrm{ppm}$, respectively. Residues for which insignificant changes of the normalized chemical shifts $(<0.04 \mathrm{ppm})$ were detected or for which no shift data could be obtained are colored in gray and white, respectively. (c) Interaction of Lck SH32 with the HMTA peptide that binds to the $\mathrm{SH} 2$ domain. Color coding as in panel a.
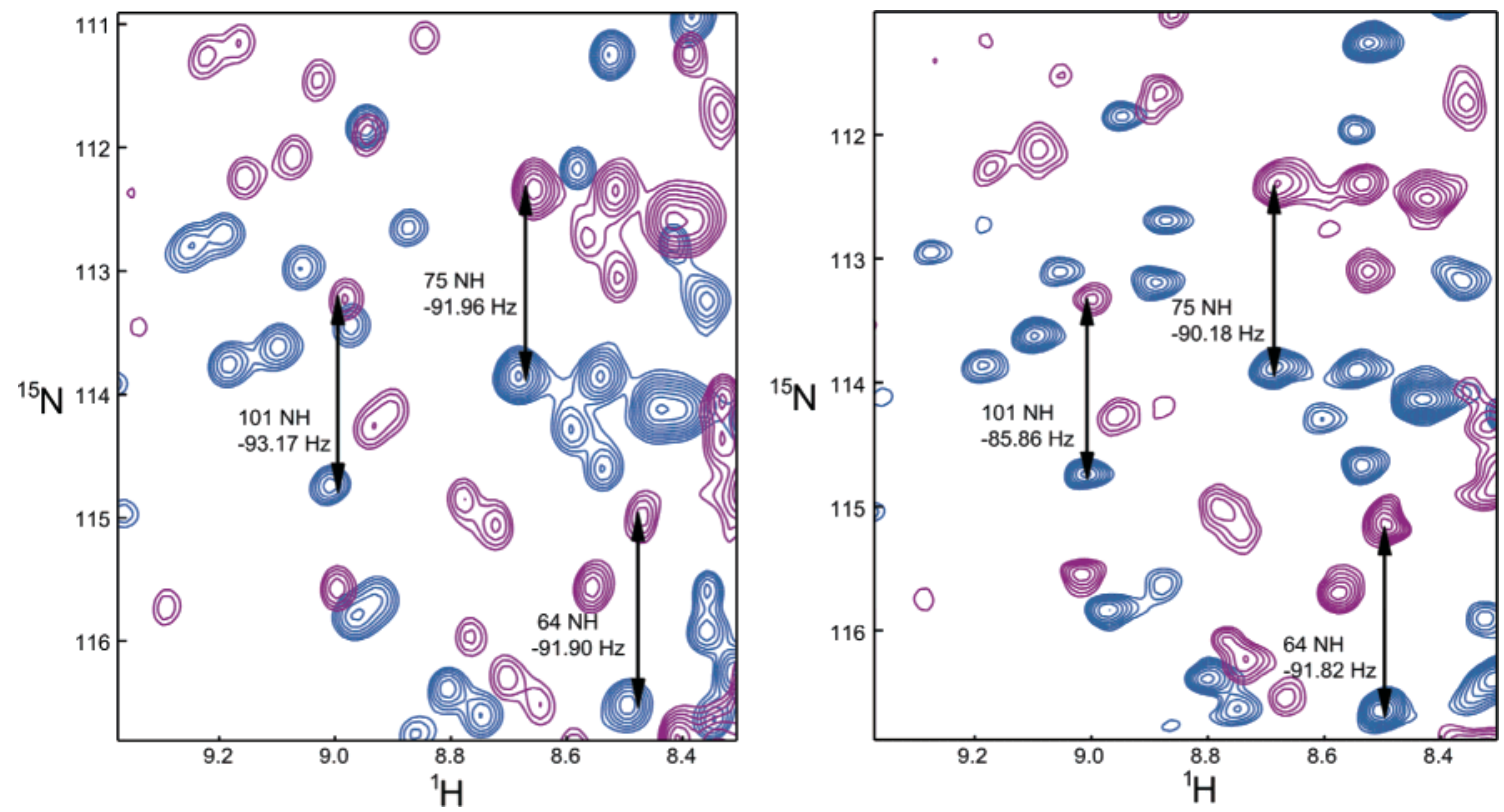

FIGURE 2: Equivalent sections of overlaid IPAP spectra of Lck SH32 in isotropic buffer solution (left) and in an aligned PAG medium (right). Some HN couplings are indicated by arrows, and their magnitude is given in hertz.

resulting in regulation pathways distinct from other members of the family.

Domain Orientation of Lck SH32 in Solution. In aqueous solution, through-space dipolar interactions are averaged to zero by isotropic tumbling and are no longer observed. However, it is possible to observe residual dipolar couplings (RDCs) in a weakly aligned medium (43). A set of such couplings provides orientational constraints that allow the determination of a preferred orientation of molecules or domains in the anisotropic medium. Comparing the alignment tensors of individual domains in domain pairs provides a sensitive measure of interdomain orientation (44).

To assess the $\mathrm{SH} 3-\mathrm{SH} 2$ domain orientation of Lck in solution, we measured its ${ }^{1} \mathrm{H}^{15} \mathrm{~N}$ RDCs (Figure 2) in an aligned polyacrylamide gel medium (PAG). Alignment tensors were then determined separately for the $\mathrm{SH} 3$ and $\mathrm{SH} 2$ domains using the published crystal structure (pdb code 1lck).
A good fit was obtained for the SH3 domain and a reasonable one for the $\mathrm{SH} 2$ domain, showing that the structure of the isolated domains is similar in solution and in the crystal. $R$ values calculated according to Clore et al. (45) were 0.23 for the SH3 domain and 0.35 for the $\mathrm{SH} 2$ domain. The worse quality of the fit for the SH2 domain is likely to be caused by structural differences induced by the presence of a phoshpotyrosine peptide bound to the $\mathrm{SH} 2$ domain in the crystal structure.

The alignment tensors corresponding to the $\mathrm{SH} 3$ and $\mathrm{SH} 2$ domains, however, have significantly different orientations suggesting that the $\mathrm{SH} 3-\mathrm{SH} 2$ domain orientation in solution is different from that found in the crystal state (Table 1). We generated model solution structures by performing rigid body rotations of the $\mathrm{SH} 2$ and $\mathrm{SH} 3$ domains that achieve alignment of the individual alignment tensors. Residue E123 in the Lck SH32 linker was chosen as a hinge for these 
Table 1: Alignment Tensors of the SH3 and SH2 Domains of Lck $\mathrm{SH} 32^{a}$

\begin{tabular}{ccccrcr}
\hline domain & $A_{\mathrm{a}}\left[10^{-5}\right]^{b}$ & $A_{\mathrm{r}}\left[10^{-5}\right]^{b}$ & $\alpha[\mathrm{deg}]^{c}$ & $\beta[\mathrm{deg}]^{c}$ & $\gamma[\mathrm{deg}]^{c}$ & $\chi_{\text {total }}^{2}$ \\
\hline SH3 & $3.7 \pm 0.4$ & $1.5 \pm 0.5$ & $112 \pm 14$ & $59 \pm 5$ & $157 \pm 5$ & 6.19 \\
SH2 & $3.8 \pm 0.5$ & $1.4 \pm 0.4$ & $149 \pm 9$ & $108 \pm 3$ & $141 \pm 5$ & 21.35
\end{tabular}

${ }^{a}$ For the fit on the Lck SH32 crystal structure 19 RDCs were included in the fit for the SH3 domain and 27 for the SH2 domain. Since the axes of the Lck SH3 and SH2 alignment tensors are not collinear, domain reorientation is necessary to obtain alignment and therefore no fit was performed for the SH32 domain pair of the crystal structure. ${ }^{b} A_{\mathrm{a}}$ and $A_{\mathrm{r}}$ are the axial and rhombic components of the alignment tensor. ${ }^{c} \alpha, \beta$, and $\gamma$ are Euler angles for the rotation of the alignment tensor into the molecular frame.

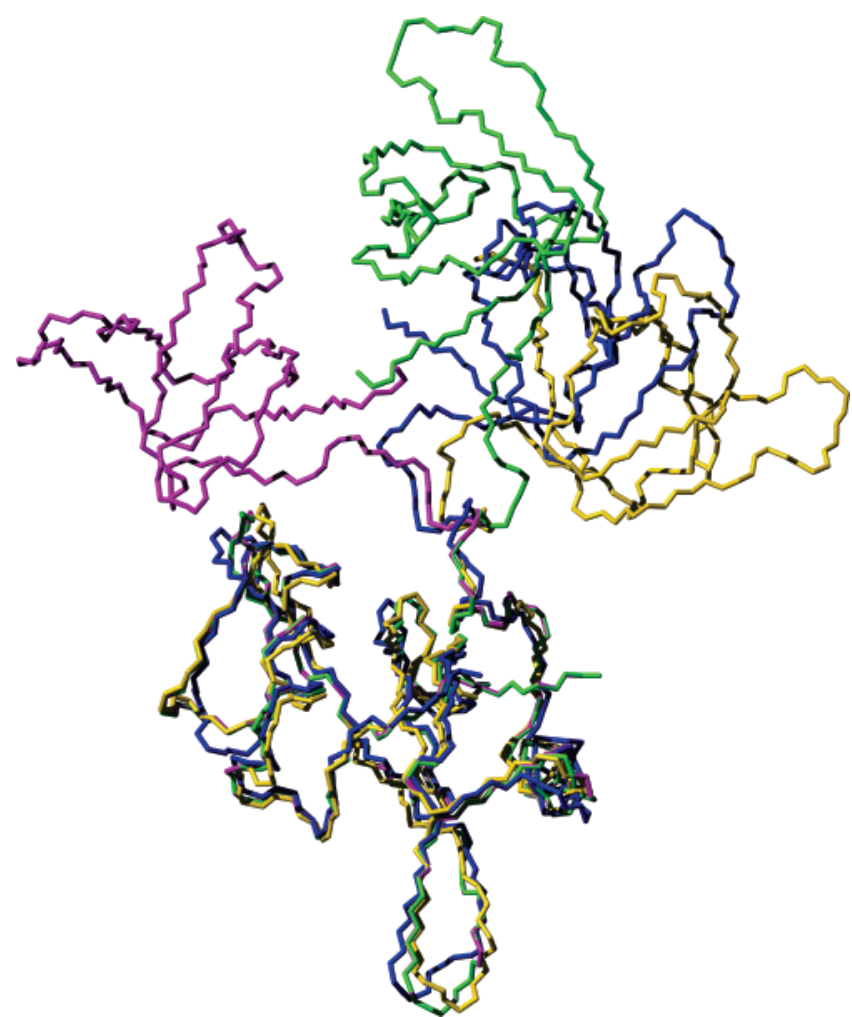

FIGURE 3: Comparison of structure of Lck-RDC (green), that is favored from combination of the data from two oriented media, with the crystal structures of Lck SH32 (magenta), Fyn SH32 (blue), and the Hck SH32 (yellow) domain pair as part of the inactive kinase. All structures are overlaid on their SH2 domain.

rotations because, in heteronuclear NOE, experiments revealed an exceptionally high flexibility (see below). Alignment tensors are degenerate with respect to $180^{\circ}$ rotations around their axes, giving rise to four different degenerate solutions. Two of these models were incompatible with the connectivity of the molecule or resulted in prohibitive steric clashes. To identify the correct model of the two remaining models a second set of RDCs in a different alignment medium was measured and subjected it to the same analysis. Comparison of the pairwise RMSDs between all the resulting models revealed that only two of the resulting orientations were reasonably close (backbone RMSD 2.6 $\AA$ ) while the others were quite different (backbone RMSD > 5.0 ̊). The structure favored from the two alignment media was termed Lck-RDC. In this model the interdomain orientations differ considerably from the orientations present in the crystal structures of either Lck SH32 or Fyn SH32, or indeed in the inactive state of Src kinases (Figure 3; Table 2).
Table 2: Summary of the Relative Domain Orientations

\begin{tabular}{lcc}
\hline \multicolumn{1}{c}{ structure } & tilt angle $^{a}[\mathrm{deg}]$ & twist $^{\text {angle }}{ }^{a}[\mathrm{deg}]$ \\
\hline Hck SH32 $^{b}$ & 13 & 306 \\
Fyn SH32 & 38 & 334 \\
Lck SH32 crystal structure & 117 & 345 \\
Lck SH32 RDC structure & 94 & 302 \\
\hline
\end{tabular}

${ }^{a}$ Tilt and twist angles for the rotation relating inertia tensors corresponding to fragments $106-109$ and 171-176 in Hck and homologous fragments in Fyn and Lck. ${ }^{b}$ As part of the Hck crystal structure of the inactive kinase.

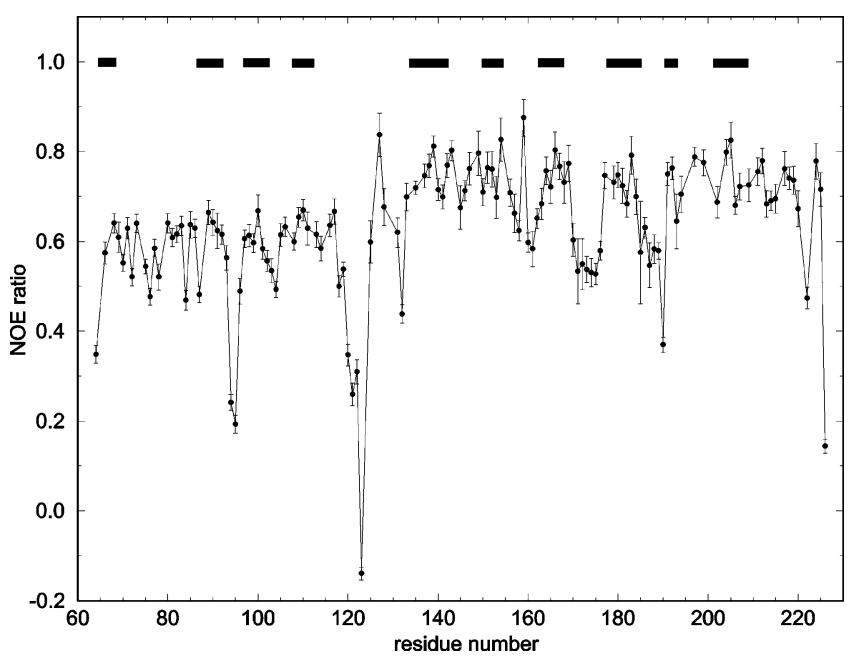

FIGURE 4: The backbone ${ }^{15} \mathrm{~N}\left\{{ }^{1} \mathrm{H}\right\}$-NOE of unbound Lck SH32 at a ${ }^{15} \mathrm{~N}$ Larmor frequency of $50 \mathrm{MHz}$. Secondary structure elements are indicated by black bars. The negative value measured for E123 which is located in the $\mathrm{SH} 3-\mathrm{SH} 2$ linker region indicates increased backbone flexibility at this sequence position.

Thus, the alignment data allowed the determination of the average relative $\mathrm{SH} 3-\mathrm{SH} 2$ domain orientation in solution, but it cannot give unambiguous information whether the two domains are mobile with respect to each other or not. The axial and rhombic components of the alignment tensor of the SH3 and SH2 domain are quite similar (Table 1), which might indicate a rigid domain orientation, but this observation alone is not sufficient to rule out interdomain dynamics. For that reason we performed a comprehensive relaxation analysis which is considered to be more sensitive for elucidating dynamic behavior of molecules.

Interdomain Dynamics of Lck SH32. NMR relaxation of nuclei in a protein in solution is mediated by rotational diffusion. Analysis of transversal $\left(T_{1}\right)$ and longitudinal $\left(T_{2}\right)$ relaxation times allows determination of characteristic tumbling times of the domains in the protein. Furthermore, the diffusion tensors to the corresponding molecules or domains can be determined reflecting the shape of the molecule. In addition, the heteronuclear $\left\{{ }^{1} \mathrm{H}\right\}{ }^{15} \mathrm{~N}$ NOE is a relaxation parameter that is particularly sensitive to local motion on a subnanosecond time scale. The lower the value for the heteronuclear $\left\{{ }^{1} \mathrm{H}\right\}{ }^{15} \mathrm{~N}$ NOE, the greater the local flexibility of the protein.

We measured the $\left\{{ }^{1} \mathrm{H}\right\}{ }^{15} \mathrm{~N}$ heteronuclear NOE for Lck SH32 to determine sites of enhanced local flexibility (Figure 4). The SH3-SH2 linker region turned out to be extremely flexible. Residue E123 has a negative NOE value indicative of a high degree of mobility. This flexible hinge region was retained in Tip-bound Lck SH32 (data not shown). Heteronuclear NOE data gathered for Fyn SH32 (11) also indicate 
a)

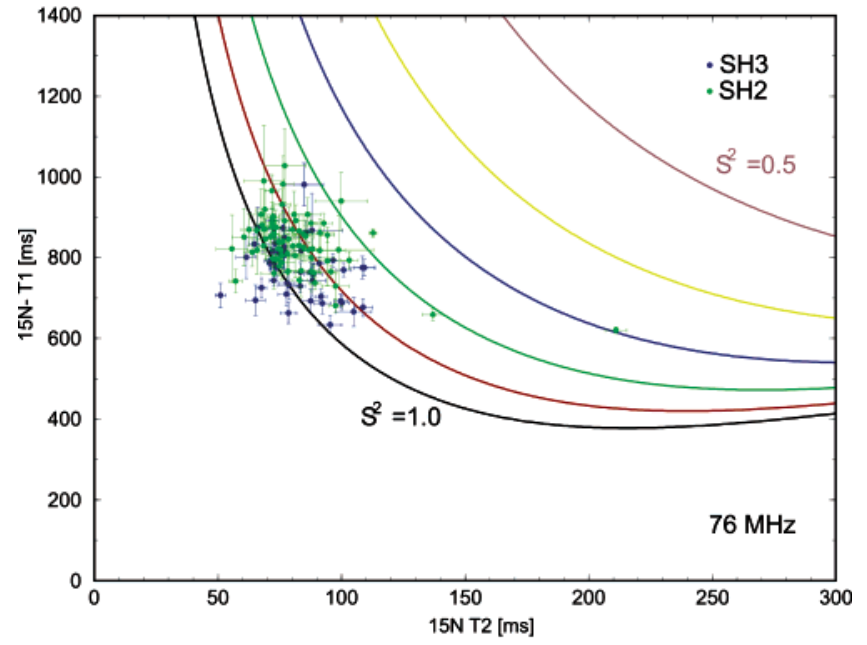

b)

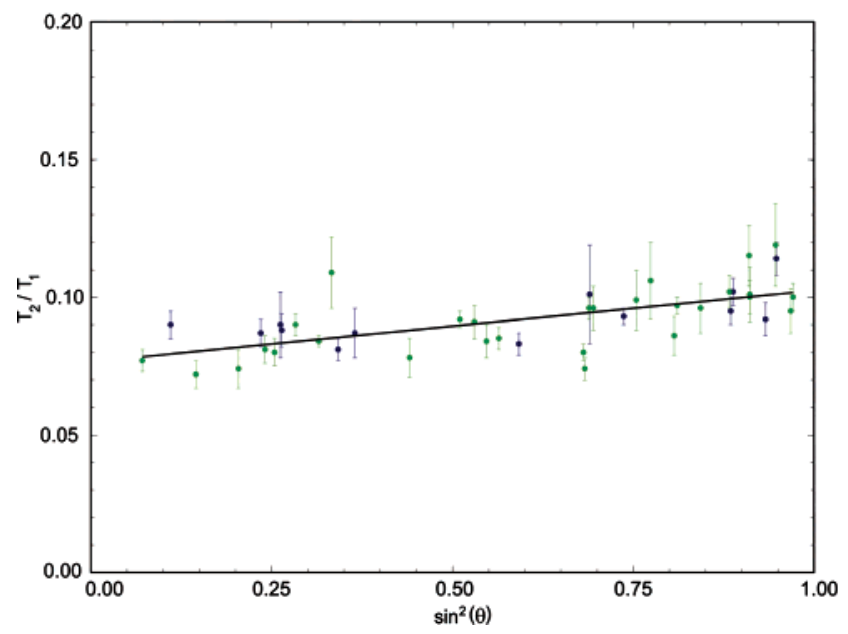

FIGURE 5: (a) ${ }^{15} \mathrm{~N} \quad T_{1}$ and $T_{2}$ relaxation times of backbone resonances of Lck SH32 at ${ }^{15} \mathrm{~N}$ Larmor frequency $76 \mathrm{MHz}$. The continuous lines define the boundaries for order parameters $S^{2}$ of the isotropic Lipari-Szabo model. As the maximum value for $S^{2}$ is 1.0, values that are outside the black boundary cannot be accounted for in terms of the simple Lipari-Szabo model and were excluded from diffusion tensor analysis. (b) Prolate diffusion tensor fit for Lck SH32. SH3 residues are shown in blue and $\mathrm{SH} 2$ ones in green. $T_{2} / T_{1}$ is plotted over $\sin ^{2}(\theta)$, where $\theta$ is the angle of the ${ }^{15} \mathrm{~N}^{1} \mathrm{H}$ bond vector fitted for each residue with respect to the principal axis of the diffusion tensor. The straight lines were obtained by linear regression, and the slope is a linear function of the axial ratio of the diffusion tensor.

a fairly flexible SH3-SH2 linker, but no negative $\left\{{ }^{1} \mathrm{H}\right\}{ }^{15} \mathrm{~N}$ NOEs were found.

${ }^{15} \mathrm{~N} T_{1}$ and $T_{2}$ relaxation time constants were determined for Lck SH32 and compared with calculated relaxation times as a function of isotropic correlation time and order parameter $\left(S^{2}\right)$ using the Lipari and Szabo model (24). For a $0.44 \mathrm{mM}$ sample of free Lck SH32 most residues were found to lie within calculated limits at $25{ }^{\circ} \mathrm{C}$ indicating the absence of aggregation (Figure 5a).

The diffusion tensors of the Lck SH32 domain pair and the individual $\mathrm{SH} 3$ and $\mathrm{SH} 2$ domains were determined using the Lck-RDC model as structural input (Table 3). The same calculations were also performed using the Lck SH32 crystal structure as input, but the fits were generally worse compared to the Lck-RDC structure (data not shown).
Treating Lck SH32 as one unit, the tumbling of the protein is best characterized by a prolate diffusion tensor with a tumbling time of $8.0 \pm 0.02 \mathrm{~ns}$ and an axial ratio of $1.32 \pm$ 0.05 . However, there are still a few residues whose $T_{1} / T_{2}$ ratio does not agree with this interpretation (Figure $5 b$ ). The reason for this can be understood by comparing the tumbling times and orientations of the diffusion tensors that are obtained for the individual domains in the Lck SH32 pair. The SH3 domain is best described by a prolate diffusion tensor with a correlation time of $7.6 \mathrm{~ns}$ and an orientation of -17 and $-1^{\circ}$ while the $\mathrm{SH} 2$ domain is best described by an asymmetric diffusion tenor with axial ratios of 1.47 and 1.24 and orientations of $21^{\circ}, 21^{\circ}$, and $3^{\circ}$ with respect to the same molecular frame (Table 3 ). These differences in correlation times and diffusion tensor orientations suggest that the domains should be treated independently. Consequently, the statistical significance of the improvement in the interpretation of the data when treating the domains independently was assessed (Table 4). According to this analysis the overall best description of the rotational diffusion of Lck SH32 treats the SH3 domains and $\mathrm{SH} 2$ as independent with a prolate diffusion tensor for the $\mathrm{SH} 3$ and an asymmetric tensor for SH2 domain. This indicates that tumbling of the $\mathrm{SH} 3$ and SH2 domains is partially decoupled and that there is a considerable degree of interdomain flexibility. This is consistent with the difference in correlation times of the individual domains $\left(\tau_{\mathrm{c}}=7.64 \pm 0.06 \mathrm{~ns}\right.$ for the $\mathrm{SH} 3$ and $8.03 \pm 0.02$ ns for the SH2 domain) and the comparatively small axial ratios (about 1.4 of the diffusion tensors) (Table $3)$. This is also consistent with the difference of the averages ${ }^{1} \mathrm{H}-{ }^{15} \mathrm{~N}-\mathrm{NOE}$ of the SH 3 and $\mathrm{SH} 2$ domains (Figure 4). The presence of significant interdomain motion in Lck SH32 means that in solution this domain pair is best described as an ensemble of many possible domain orientations. The data obtained from two orienting media, however, is not sufficient to allow quantitative statements on the relative population of different conformations within this ensemble. A qualitative analysis shows that the solution state domain orientation is roughly intermediate between that of the crystal structure of Lck SH32 and that present in the inactive state of Src kinases (Figure 3). Compared to the conformations present in the crystal structures, the $\mathrm{SH} 3-\mathrm{SH} 2$ domain interface in LckRDC is approximately $3-4$ times smaller, suggesting a decrease in stabilizing interactions. This favors a model in which the more stable conformations observed in crystal structures are also populated for Lck SH32 in solution and a conformational switching takes place between them. This suggests that the interdomain orientation as determined by RDC measurements (Figure 3) should rather be considered as a type of average orientation.

Biological Implications. In light of similarities and differences in structure and function of Lck and Fyn it is interesting to compare the present work to previous results of similar experiments performed on Fyn SH32 bound to high affinity ligands (14). Measurements on Fyn SH32 bound to either SH2 or SH3 binding ligands indicate that structural changes can be transmitted through the linker and correlate with kinase activation $(9,14)$.

In comparison Lck SH32 behaves quite differently. Lck SH32 exhibits a considerably larger interdomain flexibility compared to Fyn SH32 (14). Moreover, in Lck SH32 neither the high affinity SH2-binding ligand HMTA nor the medium 


\begin{tabular}{|c|c|c|c|c|c|c|c|c|c|}
\hline & $(6 D)^{-1}(\mathrm{~ns})$ & $2 D_{z z} /\left(D_{x x}+D_{y y}\right)$ & $D_{x x} / D_{y y}$ & $\theta^{c}$ & $\varphi^{c}$ & $\phi^{c}$ & $\chi^{2} / N^{d}$ & $Q_{1}^{e}$ & $Q_{2}{ }^{e}$ \\
\hline \multicolumn{10}{|l|}{ isotropic } \\
\hline SH32 & 8.11 & & & & & & 3.349 & & \\
\hline SH3 & 7.98 & & & & & & 2.824 & & \\
\hline SH2 & 8.14 & & & & & & 3.462 & & \\
\hline \multicolumn{10}{|c|}{$\begin{array}{l}\text { axially symmetric }{ }^{b} \\
\text { prolate }\end{array}$} \\
\hline SH32 & $8.00 \pm 0.02$ & $1.32 \pm 0.05$ & & $25.3 \pm 3.2$ & $19.8 \pm 10.4$ & & 1.775 & $6.60 \times 10^{-12}$ & \\
\hline SH3 & $7.64 \pm 0.06$ & $1.38 \pm 0.16$ & & $-17.0 \pm 9.7$ & $-1.2 \pm 18.0$ & & 0.720 & $1.40 \times 10^{-3}$ & \\
\hline SH2 & $8.03 \pm 0.02$ & $1.35 \pm 0.07$ & & $24.5 \pm 3.8$ & $21.2 \pm 12.2$ & & 1.804 & $1.07 \times 10^{-6}$ & \\
\hline \multicolumn{10}{|l|}{ oblate } \\
\hline SH32 & $8.17 \pm 0.01$ & $0.80 \pm 0.03$ & & $63.6 \pm 4.3$ & $173.6 \pm 7.1$ & & 2.556 & $2.21 \times 10^{-5}$ & \\
\hline SH3 & $7.61 \pm 0.05$ & $0.58 \pm 0.07$ & & $111.5 \pm 6.5$ & $135.3 \pm 10.0$ & & 0.848 & $3.28 \times 10^{-3}$ & \\
\hline $\mathrm{SH} 2$ & $8.09 \pm 0.02$ & $0.80 \pm 0.04$ & & $59.3 \pm 6.8$ & $161.1 \pm 10.1$ & & 2.899 & 0.11 & \\
\hline \multicolumn{10}{|l|}{ asymmetric $^{f}$} \\
\hline SH32 & 7.93 & 1.37 & 1.15 & 22.4 & 19.6 & 8.8 & 1.644 & & 0.14 \\
\hline SH3 & 7.48 & 1.46 & 1.31 & -23.5 & -8.8 & 45.4 & 0.607 & & 0.73 \\
\hline $\mathrm{SH} 2$ & 7.88 & 1.47 & 1.24 & 20.5 & 20.7 & 2.7 & 1.559 & & 0.08 \\
\hline
\end{tabular}

${ }^{a}$ Parameters were obtained by fitting experimental $T_{1} / T_{2}$ values to the Lck-RDC structure. Fits were carried out using the $T_{1} / T_{2}$ values of the residues in both the SH3 and SH2 domains, the SH3 domain only, and the SH2 domain only. ${ }^{b}$ A prolate $\left(2 D_{z z} /\left(D_{x x}+D_{y y}\right)>1\right)$ or an oblate $\left(2 D_{z z} /\left(D_{x x}+D_{y y}\right)<1\right)$ diffusion tensor was assumed. Error estimates are the standard deviations obtained by carrying out 300 Monte Carlo simulations. ${ }^{c}$ Angles describe rotations relating the molecular frame and the diffusion tensor. ${ }^{d} \chi^{2}$ values per residue. $N$ is the number of residues used for the fits. $N=42$ (SH32), 13 (SH3), 29 (SH2). ${ }^{e} Q_{1}$ was calculated for the comparison of axially symmetric diffusion tensors with the corresponding isotropic diffusion tensor. $Q_{2}$ was calculated for the comparison of asymmetric diffusion tensors with the corresponding axial diffusion tensor with lowest $\chi^{2} \cdot{ }^{f}$ No error estimates could be determined for the parameters of asymmetric diffusion tensor

Table 4: Comparison of Selected Diffusion Tensor Models

\begin{tabular}{clcccc}
\hline no. & \multicolumn{1}{c}{ Lck-RDC $^{a}$} & $\chi^{2} /$ res $^{b}$ & $Q_{1}{ }^{c}$ & $Q_{2}{ }^{c}$ & $Q_{3}{ }^{c}$ \\
\hline 1 & SH32 prolate & 1.8 & & & \\
2 & SH3 prolate & 0.7 & & & \\
3 & SH2 prolate & 1.8 & & & \\
4 & SH2 asymmetric & 1.6 & 0.08 & & \\
5 & SH3-2 pr/pr & 1.5 & & $4.72 \times 10^{-2}$ & \\
6 & SH3-2 $\mathrm{pr}^{d}$ as $^{e}$ & 1.3 & & $2.81 \times 10^{-2}$ & $2.00 \times 10^{-2}$ \\
\hline
\end{tabular}

${ }^{a}$ Name of the structure, as used in the text. ${ }^{b}$ The total number of residues included in the analysis was 42 for models 1,5 , and $6 ; 13$ for model 2; and 29 for models 3 and $4 .{ }^{c} Q_{1}$ compares models 3 and 4 ; $Q_{2}$ compares models 5 and 6 with model 1 ; and $Q_{3}$ compares model 5 with model $6 .{ }^{d}$ Combination of independent prolate diffusion tensors for the $\mathrm{SH} 2$ and $\mathrm{SH} 3$ domains. ${ }^{e}$ Combination of a prolate diffusion tensor for the SH3 domain and an asymmetric diffusion tensor for the $\mathrm{SH} 2$ domain.

affinity SH3-binding ligand Tip(167-199) induced any chemical shifts in the adjacent domain despite being able to activate Lck $(34,42)$.

The greater flexibility of the linker region in Lck SH32 as compared to Fyn SH32 (14), and the absence of interdomain coupling, can be rationalized by the different composition of its linker. Lck has a proline-X-proline motif in the SH2-SH3 linker region, while Src, Hck, and Fyn contain a glutamate in the linker that can form electrostatic interactions with a lysine of the SH3 domain (11), thus stabilizing a linker conformation that is compatible with a domain orientation close to that found in the inactive state of Src kinases.

Considered on its own, the relative flexibility of the Lck SH32 domain pair would also be expected to shift the equilibrium between the active and inactive state of Lck in favor of the active state. This is of course only one of many factors contributing to the finely tuned control of this allosteric switch.

Fyn and Lck are both expressed in T-lymphocytes and share a pairwise sequence identity of $54 \%$. While Lck expression is essentially confined to this type of cell, Fyn is expressed in a broader range of cells, taking part in diverse signaling pathways. Both have been implicated in T-cell activation and differentiation, and it has been suggested that Fyn can act as a backup for Lck to some extent (3). Nonetheless, some functions have been identified that are unique to Lck. Examples are the positive selection of CD4 single-positive cells and the proliferation of T-cells in lymphopenic environments (46). The significant differences in SH2-SH3 linkage observed here may help to adapt Lck and Fyn to their specific surroundings and to achieve their functional differences.

\section{ACKNOWLEDGMENT}

The authors thank Sabine Schwarz for helpful discussion on the manuscript.

\section{SUPPORTING INFORMATION AVAILABLE}

One figure showing an overlay of the domain orientations determined from the two anisotropic media and one figure showing an overlay of the best-fitting diffusion tensors mapped on the Lck SH32 structure. This material is available free of charge via the Internet at http://pubs.acs.org.

\section{REFERENCES}

1. Bolen, J. B. (1993) Nonreceptor tyrosine protein kinases, Oncogene 8, 2025-2031.

2. Brown, M. T., and Cooper, J. A. (1996) Regulation, substrates and functions of src, Biochim. Biophys. Acta 1287, 121-149.

3. Thomas, S. M., and Brugge, J. S. (1997) Cellular functions regulated by Src family kinases, Annu. Rev. Cell Dev. Biol. 13, 513-609.

4. Williams, J. C., Weijland, A., Gonfloni, S., Thompson, A., Courtneidge, S. A., Superti-Furga, G., and Wierenga, R. K. (1997) The 2.35 A crystal structure of the inactivated form of chicken Src: a dynamic molecule with multiple regulatory interactions, J. Mol. Biol. 274, 757-775.

5. Xu, W., Harrison, S. C., and Eck, M. J. (1997) Three-dimensional structure of the tyrosine kinase c-Src, Nature 385, 595-602.

6. Sicheri, F., Moarefi, I., and Kuriyan, J. (1997) Crystal structure of the Src family tyrosine kinase Hck, Nature 385, 602-609. 
7. Mustelin, T., and Altman, A. (1990) Dephosphorylation and activation of the $\mathrm{T}$ cell tyrosine kinase pp56lck by the leukocyte common antigen (CD45), Oncogene 5, 809-813.

8. Moarefi, I., LaFevre-Bernt, M., Sicheri, F., Huse, M., Lee, C. H., Kuriyan, J., and Miller, W. T. (1997) Activation of the Src-family tyrosine kinase Hck by SH3 domain displacement, Nature 385, 650-653.

9. Young, M. A., Gonfloni, S., Superti-Furga, G., Roux, B., and Kuriyan, J. (2001) Dynamic coupling between the SH2 and SH3 domains of $\mathrm{c}-\mathrm{Src}$ and Hck underlies their inactivation by $\mathrm{C}$ terminal tyrosine phosphorylation, Cell 105, 115-126.

10. Eck, M. J., Atwell, S. K., Shoelson, S. E., and Harrison, S. C. (1994) Structure of the regulatory domains of the Src-family tyrosine kinase Lck, Nature 368, 764-769.

11. Arold, S. T., Ulmer, T. S., Mulhern, T. D., Werner, J. M., Ladbury, J. E., Campbell, I. D., and Noble, M. E. (2001) The role of the Src homology 3-Src homology 2 interface in the regulation of Src kinases, J. Biol. Chem. 276, 17199-17205.

12. Tessari, M., Gentile, L. N., Taylor, S. J., Shalloway, D. I., Nicholson, L. K., and Vuister, G. W. (1997) Heteronuclear NMR studies of the combined Src homology domains 2 and 3 of pp60 c-Src: effects of phosphopeptide binding, Biochemistry 36, $14561-14571$.

13. Fushman, D., Xu, R., and Cowburn, D. (1999) Direct determination of changes of interdomain orientation on ligation: use of the orientational dependence of $15 \mathrm{~N}$ NMR relaxation in $\mathrm{Abl} \mathrm{SH(32),}$ Biochemistry 38, 10225-10230.

14. Ulmer, T. S., Werner, J. M., and Campbell, I. D. (2002) SH3SH2 domain orientation in Src kinases: NMR studies of Fyn, Structure 10, 901-911.

15. Schweimer, K., Kiessling, A., Bauer, F., Hor, S., Hoffmann, S., Rosch, P., and Sticht, H. (2003) Sequence-specific 1H, 13C and $15 \mathrm{~N}$ resonance assignments of the $\mathrm{SH} 3-\mathrm{SH} 2$ domain pair from the human tyrosine kinase Lck, J. Biomol. NMR 27, 405406.

16. Chou, J. J., Gaemers, S., Howder, B., Louis, J. M., and Bax, A. (2001) A simple apparatus for generating stretched polyacrylamide gels, yielding uniform alignment of proteins and detergent micelles, J. Biomol. NMR 21, 377-382.

17. Ruckert, M., and Otting, G. (2000) Alignment of biological macromolecules in novel nonionic liquid crystalline media for NMR experiments, J. Am. Chem. Soc. 122, 7793-7797.

18. Kay, L. E., Torchia, D. A., and Bax, A. (1989) Backbone dynamics of proteins as studied by $15 \mathrm{~N}$ inverse detected heteronuclear NMR spectroscopy: application to staphylococcal nuclease, Biochemistry $28,8972-8979$.

19. Kay, L. E., Keifer, P., and Saarinen, T. (1992) Pure absorption gradient enhanced heteronuclear single quantum correlation spectroscopy with improved sensitivity, J. Am. Chem. Soc. 114, 10663-10665.

20. Farrow, N. A., Zhang, O., Forman-Kay, J. D., and Kay, L. E. (1994) A heteronuclear correlation experiment for simultaneous determination of $15 \mathrm{~N}$ longitudinal decay and chemical exchange rates of systems in slow equilibrium, J. Biomol. NMR 4, 727734.

21. Boyd, J., Hommel, U., and Campbell, I. D. (1990) Influence Of Cross-Correlation Between Dipolar and Anisotropic Chemical Shift Relaxation Mechanisms Upon Longitudinal Relaation Rates Of N-15 in Macromolecules., Chem. Phys. Lett. 175, 477482.

22. Kay, L. E., Nicholson, L. K., Delaglio, F., and Bax, A. (1992) Pulse Sequences for Removal of the Effects of Cross-Correlation between Dipolar and Chemical-Shift Anisotropy Relaxation Mechanism on the Measurement of Heteronuclear T1 and T2 Values in Proteins, J. Magn. Reson. 97, 359-375.

23. Werner, J. M., Campbell, I. D., and Downing, A. K. (2002) Shape and dynamics of a calcium-binding protein investigated by nitrogen-15 NMR relaxation, Methods Mol. Biol. 173, 285-300.

24. Lipari, G., and Szabo, A. (1982) Model-free approach to the investigation of nuclear magnetic resonance relaxation in macromolecules, J. Am. Chem. Soc. 104, 4546-4559.

25. Palmer, A. G., Rance, M., and Wright, P. E. (1991) Intramolecular motions of a zinc finger DNA-binding domain from xfin characterized by proton-detected natural abundance $12 \mathrm{C}$ heteronuclear NMR-spectroscopy, J. Am. Chem. Soc. 113, 43714380.

26. Mandel, A. M., Akke, M., and Palmer, A. G., 3rd. (1995) Backbone dynamics of Escherichia coli ribonuclease HI: cor- relations with structure and function in an active enzyme, J. Mol. Biol. 246, 144-163.

27. Boyd, J., and Redfield, C. (1998) Defining the orientation of the $\mathrm{N}-15$ shielding tensor using N-15 NMR relaxation data for a protein in solution, J. Am. Chem. Soc. 120, 9692-9693.

28. Woessner, D. E. (1962) Nuclear spin relaxation in ellipsoids undergoing rotational Brownian motion, J. Chem. Phys. 37, 647654.

29. Press, W. H., Flannery, B. P., Teukosky, S. A., and Vetterling, W. T. (1990) Numerical Recipes, Cambridge University Press, New York.

30. Ottiger, M., Delaglio, F., and Bax, A. (1998) Measurement of J and dipolar couplings from simplified two-dimensional NMR spectra, J. Magn. Reson. 131, 373-378.

31. Dosset, P., Hus, J. C., Marion, D., and Blackledge, M. (2001) A novel interactive tool for rigid-body modeling of multi-domain macromolecules using residual dipolar couplings, J. Biomol. NMR 20, 223-231.

32. Morton, C. J., Pugh, D. J., Brown, E. L., Kahmann, J. D., Renzoni, D. A., and Campbell, I. D. (1996) Solution structure and peptide binding of the SH3 domain from human Fyn, Structure 4, 705714.

33. Schweimer, K., Hoffmann, S., Bauer, F., Friedrich, U., Kardinal, C., Feller, S. M., Biesinger, B., and Sticht, H. (2002) Structural investigation of the binding of a herpesviral protein to the $\mathrm{SH} 3$ domain of tyrosine kinase Lck, Biochemistry 41, 5120-5130.

34. Hartley, D. A., Amdjadi, K., Hurley, T. R., Lund, T. C., Medveczky, P. G., and Sefton, B. M. (2000) Activation of the Lck tyrosine protein kinase by the Herpesvirus saimiri tip protein involves two binding interactions, Virology 276, 339-348.

35. Wiese, N., Tsygankov, A. Y., Klauenberg, U., Bolen, J. B., Fleischer, B., and Broker, B. M. (1996) Selective activation of T cell kinase p56lck by Herpesvirus saimiri protein tip, J. Biol. Chem. 271, 847-852.

36. Songyang, Z., Shoelson, S. E., Chaudhuri, M., Gish, G., Pawson, T., Haser, W. G., King, F., Roberts, T., Ratnofsky, S., Lechleider, R. J., et al. (1993) SH2 domains recognize specific phosphopeptide sequences, Cell 72, 767-778.

37. Morelock, M. M., Ingraham, R. H., Betageri, R., and Jakes, S. (1995) Determination of receptor-ligand kinetic and equilibrium binding constants using surface plasmon resonance: application to the lck SH2 domain and phosphotyrosyl peptides, J. Med. Chem. $38,1309-1318$.

38. Bauer, F., Hofinger, E., Hoffmann, S., Rosch, P., Schweimer, K., and Sticht, H. (2004) Characterization of Lck-binding elements in the herpesviral regulatory Tip protein, Biochemistry 43, 1493214939.

39. Courtneidge, S. A. (1986) Transformation by polyoma virus middle T antigen, Cancer Surv. 5, 173-182.

40. Kaplan, D. R., Pallas, D. C., Morgan, W., Schaffhausen, B., and Roberts, T. M. (1989) Mechanisms of transformation by polyoma virus middle T antigen, Biochim. Biophys. Acta 948, 345-364.

41. Dunant, N. M., Messerschmitt, A. S., and Ballmer-Hofer, K. (1997) Functional interaction between the SH2 domain of Fyn and tyrosine 324 of hamster polyomavirus middle-T antigen, J. Virol. 71, 199-206.

42. Pelosi, M., Di Bartolo, V., Mounier, V., Mege, D., Pascussi, J. M., Dufour, E., Blondel, A., and Acuto, O. (1999) Tyrosine 319 in the interdomain B of ZAP-70 is a binding site for the Src homology 2 domain of Lck, J. Biol. Chem. 274, 1422914237.

43. Tjandra, N., and Bax, A. (1997) Direct measurement of distances and angles in biomolecules by NMR in a dilute liquid crystalline medium, Science 278, 1111-1114

44. Losonczi, J. A., Andrec, M., Fischer, M. F. W., and Prestegard, J. H. (1999) Order matrix analysis of residual dipolar couplings using singular value decomposition, J. Magn. Reson. 138, 334342

45. Clore, G. M., and Garrett, D. S. (1999) Free R and Complete CrossValidation for Dipolar Coupling Refinement of NMR Structures. J. Am. Chem. Soc. 121, 9008-9012.

46. Zamoyska, R., Basson, A., Filby, A., Legname, G., Lovatt, M., and Seddon, B. (2003) The influence of the src-family kinases, Lck and Fyn, on T cell differentiation, survival and activation, Immunol. Rev. 191, 107-118.

BI050814Y 